\title{
Cirugía transnasal transesfenoidal endoscópica en afecciones de región selar
}

\author{
J.L. González-González; O. López-Arbolay; O. Morales-Sabina; J.F. Martí-Piñeiro y R. Vidal-Verdial
}

Servicio de Neurocirugía. Hospital Hermanos Ameijeiras. Ciudad de la Habana. Cuba.

Resumen

Introducción. Para un gran número de tumores pituitarios la cirugía es el tratamiento de primera línea y el abordaje transesfenoidal microquirúrgico el más utilizado. En estos momentos, el mismo se ejecuta tanto por la vía sublabial como por la transnasal. Recientemente, el abordaje transnasal endoscópico ha irrumpido como una opción en desarrollo, que pudiera llegar a predominar en un futuro próximo.

Objetivos. Evaluar mediante el análisis de los resultados si es posible realizar en nuestro medio el abordaje transnasal transesfenoidal endoscópico para las lesiones de la región selar con resultados quirúrgicos, al menos, similares a los obtenidos con el método transesfenoidal tradicional.

Material y método. Como fase I de un ensayo en 3 etapas se realiza una investigación limitada tipo cohorte, estrictamente controlada y prospectiva de 10 pacientes seleccionados según criterios de inclusión y a los cuales se les practicó el abordaje transnasal endoscópico como método de tratamiento quirúrgico entre Agosto del 2002 y Agosto del 2003 en el servicio de Neurocirugía del Hospital "Hermanos Ameijeiras".

Resultados. Se operaron seis casos que tenían macroadenomas hipofisarios, tres productores de $\mathbf{G H}$, uno secretor de PRL y otros dos no productores. A los otros cuatro se les había diagnosticado fístula de LCR a través del piso selar. De los seis macroadenomas, en cinco se logró exéresis total del tumor, mientras que en el otro se obtuvo exéresis subtotal. La rinorrea desapareció en 2 pacientes con fístula de LCR y se mantuvo en los otros 2. De las complicaciones observadas en el $30 \%$ de los casos, solo en $1(10 \%)$ transcendieron más allá del transoperatorio.

Conclusiones. Los resultados obtenidos en la fase 1 con la utilización del método transnasal endoscópico, hacen que el procedimiento en nuestro medió sea lo suficientemente seguro como para justificar el pase a la

Recibido: 15-03-04. Aceptado: 20-04-04 fase II del ensayo (aleatorizada y controlada), en busca de información más consistente, en relación al valor real de la técnica a estas alturas del desarrollo de la cirugía hipofisaria.

PALABRAS CLAVE: Abordaje. Adenomectomía. Endoscópica. Fístulas. Macroadenomas. Transnasal. Transesfenoidal.

Transnasal-transsphenoidal endoscopic surgery of the sellar region

\section{Summary}

Transnasal transsphenoidal endoscopic approach to the sella turcica has recently become an option to the pituitary surgery which could predominate in a near future.

Objectives. To evaluate through the analysis of our results whether it is possible to perform in our Hospital the endoscopic transnasal transsphenoidal approach to the sella with results at least as good as those we obtain with the microscopic sublabial transseptal transsphenoidal approach.

Material and methods. As the first stage of a three stage randomized trial, this is a prospective study of $\mathbf{1 0}$ patients operated on, using transnasal transsphenoidal endoscopic aproach between august 2002 and August 2003 at the department of neurosurgery of "Hermanos Ameijeiras Hospital".

Results. Six patients had pituitary macroadenomas and four had cerebrospinal fluid leak through the sellar floor. In five of six macroadenomas total tumor resection was obtained and in the other one a subtotal resection was performed. Two of four patients with cerebrospinal fluid leak could be cured while the other two patients do not. Complications were present in $30 \%$ of cases, but in only $1(\mathbf{1 0 \%})$ it reached the postoperatory period.

Conclusions. Transnasal transsphenoidal endoscopic approach to the sella can be done save enough in our Hospital to justify the start of the second stage of one 
randomized trial to determine if the endoscopic technique is better than the usual transsphenoidal approach.

KEY WORDS: Approach. Pituitary. Endoscopic. Transnasal. Transsphenoidal.

\section{Introducción}

Las afecciones de la región selar son frecuentes en la práctica neuroquirúrgica. Los tumores de esta región suman entre el 10 y el $15 \%$ de las neoplasias intracraneales ${ }^{10,24}$. La incidencia, en series de autopsia alcanza hasta un $25 \%$ de la población ${ }^{11,32}$. En orden decreciente, los adenomas hipofisarios son el tercer tipo de tumor primario del sistema nervioso central ${ }^{1,17}$. Para un gran número de tumores pituitarios la cirugía es el tratamiento de primera línea, debido a la respuesta rápida, y con frecuencia definitiva, que muchas veces se logra ${ }^{17}$.

Las fístulas de líquido cefalorraquídeo (1.c.r.) a través del piso selar consecutivas a la cirugía o espontáneas (casi siempre en relación con silla vacía), tampoco son infrecuentes en la práctica neuroquirúrgica. Tanto para fístulas como para adenomas, el abordaje transesfenoidal ha sido, desde la segunda mitad del pasado siglo, la alternativa de solución más versátil y utilizada, alcanzando más del 95\% de las operaciones cuyo blanco es la silla $\operatorname{turca}^{16,17}$.

El uso del endoscopio en la cirugía pituitaria a través de los senos paranasales fue reportado por vez primera en los años 1970 en la Literatura médica alemana ${ }^{3,13,22}$. En 1989, Papay ${ }^{26}$ emplea el abordaje transeptal endoscópico para reparar fístulas de 1.c.r. secundarias a cirugía pituitaria y en 1992, Jankowski reporta la resección endonasal endoscópica exitosa de adenomas hipofisarios en tres pacientes ${ }^{18}$. Dos años después, Gamea expone su experiencia en 10 casos de tumores hipofisarios abordados por vía sublabial transeptal transesfenoidal utilizando el microscopio quirúrgico y apoyándose con el endoscopio. Este autor concluyó que el endoscopio facilitaba la disección. del tumor de la glándula normal ${ }^{12}$.

En 1996, Sethi publica una serie de 40 pacientes tratados consecutivamente mediante la aplicación del abordaje transnasal endoscópico para el tratamiento de adenomas hipofisarios y craneofaringiomas ${ }^{30} \mathrm{y}$ en el mismo año y el siguiente Jho y Carrau publicaron en sendos artículos, su experiencia inicial primero y luego en 50 pacientes, con ayuda de otro cirujano que sostiene el endoscopio después de la esfenoidotomía y le permite la operación bimanual. Inmediatamente reportan la introducción de un soporte mecánico con este fin ${ }^{6,20,21}$.

Según Rodziewicz y Heilman, la descompresión endoscópica de los tumores pituitarios puede realizarse alcanzando buenos resultados con mínima morbilidad qui- rúrgica $^{15,29}$.

Aldo Stamm señala varias ventajas de la cirugía pituitaria endonasal endoscópica. La primera y más importante es proveer un acceso más directo y rápido a la silla sin craneotomía, lo que reduce la morbilidad asociada a este tipo de proceder. También mejora la visualización, el ángulo visual, y amplía la perspectiva panorámica de importantes estructuras anatómicas del seno esfenoidal, silla turca y región paraselar, permitiendo un manejo dinámico y con seguridad durante la cirugía ${ }^{32}$.

Algunos autores han llegado a plantear que el endoscopio ha reemplazado al microscopio quirúrgico en la cirugía de los adenomas hipofisarios ${ }^{7,12,18,20,23,25,30,31}$. El aporte del grupo de la Universidad Federico II de Nápoles, trabajando sobre la anatomía endoscópica y el diseño de instrumentos adecuados para esta cirugía, ha sido sustancial con vistas a convertirla en una técnica estándar.

Como quiera que estamos hablando de un procedimiento aun de reciente aparición en la especialidad, el servicio de Neurocirugía del Hospital "Hermanos Ameijeiras", donde cada año se realiza la mayor cifra de operaciones a afecciones selares en el país, ha diseñado un ensayo para comparar los resultados de la cirugía transnasal endoscópica con los de la microcirugía transeptoesfenoidal, con el objetivo de aclarar las interrogantes existentes en relación con la eficacia y morbimortalidad del método endoscópico y analizar la conveniencia o no de su aplicación sistemática. En este artículo exponemos los resultados de la Fase I del mencionado estudio.

\section{Pacientes y método}

\section{Tipo de estudio}

I: Ensayo preliminar o estudio corto. Se realiza una investigación limitada tipo cohorte, estrictamente controlada y prospectiva de 10 pacientes seleccionados según los criterios de inclusión y a los cuales se les realizó el abordaje transnasal endoscópico como método de tratamiento quirúrgico para su enfermedad en cuestión entre agosto del 2002 y agosto del 2003, con vistas a comprobar la factibilidad en nuestro medio de la ejecución de dicha técnica.

\section{Los criterios de inclusión fueron:}

- Adenomas hipofisiarios con crecimiento intraselar hasta $10 \mathrm{~mm}$ o supraselar hasta cisterna quiasmática, respetando el III ventrículo.

- Fístulas de 1.c.r. a través del piso selar al seno esfenoidal comprobadas por resonancia magnético nuclear (RMN) y/o o por endoscopía de la cavidad nasal.

\section{Criterios de exclusión: \\ - Microprolactinomas $(<10 \mathrm{~mm})$. \\ - Microadenomas no secretores.}


-No aceptación por parte del paciente.

Se entrevistó a cada uno de los pacientes que reunieron los criterios de inclusión, a los cuales se les explicaron los objetivos del estudio, así como los aspectos generales de cada procedimiento (transeptoesfenoidal y transnasal endoscópica) de forma clara y comprensible, después de lo cual se solicito su consentimiento para ser incluido en el ensayo. En los casos en que el paciente estuvo de acuerdo, se le pidió que llenara y firmara un modelo de consentimiento informado y, sólo después de esto, fue elegido para el procedimiento endoscópico.

El corte en el seguimiento de los pacientes fue a los tres meses posteriores a la cirugía con la evaluación clínica y los estudios complementarios necesarios, según la enfermedad de cada caso.

Las fases II y III de la investigación están en marcha y sus resultados se presentarán en futuros artículos.

Fase II: estudio controlado, a simple ciego, en el cual se le ofrecerá a los pacientes de forma aleatoria, previo consentimiento informado, el abordaje transnasal endoscópico o el abordaje sublabial transeptal microquirúrgico como método de tratamiento para su enfermedad.

Fase III: será la fase de seguimiento con un corte a los 6 meses posteriores a la cirugía, con evaluación clínica y estudios complementarios, según protocolo para la afección en cuestión.

La técnica quirúrgica empleada fue básicamente la descrita previamente por Jho y Carrau ${ }^{20}$, aunque en los primeros 6 pacientes, se realizó turbinectomía media parcial, con vistas a ampliar la cavidad nasal y facilitar el proceder. En los cuatro restantes este paso fue abandonado. Se utilizaron dos endoscopios rígidos de $30 \mathrm{~cm}$ de longitud. Uno de ellos de $2,7 \mathrm{~mm}$ de diámetro y lente de $30^{\circ}$. El otro de $4 \mathrm{~mm}$ y lente de $0^{\circ}$. El primero de ellos se utilizó dentro de una vaina para facilitar la aspiración y lavado del lente.

\section{Resultados}

Los resultados generales están reflejados en la tabla 1 .

Nueve pacientes fueron del sexo femenino y uno del masculino. Las edades oscilaron entre los 19 y 61 años.

El diagnóstico fue macroadenoma hipofisario en 6 casos, tres de ellos productores de hormona del crecimiento (GH) (casos 1, 2 y 8), uno secretor de prolactina (PRL) (caso 9) y los otros dos no secretores (casos 3 y 10). Los otros cuatro $(4,5,6$ y 7$)$ tenían fístula de 1.c.r. a través del piso selar.

Entre los principales síntomas observados, la cefalea estuvo presente en 8 casos (80\%). El resto fueron variados, en concordancia con la de entidad de cada paciente. Los 3 pacientes con adenomas productores de GH presentaron crecimiento acral (acromegalia). La paciente con diagnóstico de prolactinoma presentó amenorrea y galactorrea
(Síndrome de Forbes-Albright). Los 2 casos con adenomas no secretores debutaron con apoplejía pituitaria (cefalea y defecto campimétrico de instalación súbita) y hemorragia subaracnoidea (cefalea intensa, inconsciencia transitoria y parálisis unilateral del III) respectivamente. Los cuatro pacientes con fístula de LCR presentaban rinorrea y, excepto uno, aquejaban cefalea.

En el postoperatorio, la cefalea desapareció en $4(80 \%)$ y mejoró en $1(20 \%)$ de los 5 pacientes con adenomas que previamente la padecían, persistiendo en $2(66,7 \%)$, (precisamente aquellos que no resolvieron la fístula con la operación) y mejorando en $1(33,3 \%)$ de los 3 pacientes con fístula de LCR que ya la presentaban. El crecimiento acral se detuvo y tendió a la regresión en los tres casos con acromegalia. En la paciente con macroprolactinoma (caso 9), desapareció la galactorrea y reaparecieron las menstruaciones. El paciente con trastornos del campo visual (caso 3), mostró desaparición de los mismos, después de la cirugía y en la paciente con oftalmoplejia del III nervio craneal (caso 10), ésta desapareció al mes de operada.

La rinorrea desapareció en 2 pacientes con fístula de LCR $(50 \%)$ y se mantuvo igual en los otros $2(50 \%)$.

De los tres macroadenomas secretores de $\mathrm{GH}$, en uno $(33,3 \%)$ se consiguió reducir el valor de esta hormona a la mitad del valor inicial (caso 1) y en los otros dos $(66,6 \%)$ se redujo a cifras normales $(<5 \mathrm{ng} / \mathrm{ml})$. La paciente con macroadenoma secretor de PRL normalizó la cifra de esta hormona. En los no productores no hubo variaciones hormonales.

La evaluación con tomografía axial computada en el postoperatorio inmediato y a los 3 meses, arrojo que en cinco de los seis macroadenomas se logró la exéresis total del tumor $(83.3 \%)$, mientras que en uno esta fue subtotal (reducción del volumen tumoral del 80\%).

De acuerdo a la evaluación por RMN de los cuatro pacientes con fístula de LCR a través del piso selar, en dos se obtuvo desaparición del trayecto fistuloso $(50 \%)$ y en dos no se consiguió.

Algún evento que pudo ser considerado como complicación propia del procedimiento se observó en el 30\% de los casos. Sólo en uno de ellos (10\%) ésta trascendió más allá del transoperatorio y resulto ser una meningoencefalitis bacteriana (caso 4).

La estancia hospitalaria postoperatoria fluctuó entre dos y diecisiete días, con una media de ocho.

El tiempo quirúrgico promedio fue de tres horas cuarenta minutos y varió desde dos hasta seis horas.

\section{Discusión}

\section{Abordaje}

El abordaje endoscópico a la región selar nació desde 
propuestas diferentes. Reportes iniciales de Papay y colaboradores defienden el abordaje transnasal transeptal endoscópico sobre el nasal endoscópico e incluso sobre el microquirúrgico ${ }^{26}$. Este autor realiza toda la intervención a través de uno de los lados de la nariz. Para Rodziewics, sin embargo, aunque también de forma submucosa, resulta ventajoso ejecutar la operación introduciendo el endoscopio a través de una incisión de $8 \mathrm{~mm}$ a la izquierda y los instrumentos por otro túnel submucoso derecho ${ }^{29}$, evitando así interferencias entre ellos. Ambas variantes llevan implícitas disección mucoseptal y posibilidad de sinequias, cambios estéticos y mayor grado de molestias postoperatorias. Sólo la falta de desinserción del tabique las diferencia físicamente del proceder microquirúrgico clásico. Se han llevado a cabo bastantes intervenciones en recientes series, de forma totalmente transnasal y unilateral con buenos resultados; se evitaba el trauma y consumo de tiempo propios de la disección septal ${ }^{4,21}$. Consideramos estas ventajas lo suficientemente consistentes como para adherirnos a esta última variante.

Se ha recomendado la resección parcial del cornete medio y ocasionalmente del superior para dar amplitud a una fosa nasal y de esta forma facilitar el paso de endoscopio e instrumentos y mejorar la visibilidad ${ }^{18,32}$. Aunque esto fue así en los primeros 6 pacientes de la presente serie, pronto coincidimos con los que afirman que este detalle es innecesario si se siguen determinadas reglas, como la elección de la fosa nasal de mayor amplitud (teniendo en cuenta la lateralización del tabique, desarrollo de los cornetes, presencia de una concha bulosa) y la penetración gradual del endoscopio junto al piso nasal hasta la identificación de los cornetes inferior y luego medio, para entonces ascender ligeramente entre éste y el tabique nasal y avanzar hasta visualizar el "rostrum" y "ostium" del esfenoides ${ }^{4,20}$. Por tal motivo abandonamos esta práctica sin percibir por ello un incremento en el grado de dificultad técnica para el abordaje.

\section{Visibilidad}

Si bien es cierto que el microscopio ofrece una visión estereoscópica y familiar del campo quirúrgico, la bidimensionalidad de la visión endoscópica se ve compensada por el acercamiento directo de ésta a la anatomía de la región, lo que permite una mejor definición de las interfases tumor-tejido glandular y glándula-diafragma/aracnoides. Este acercamiento y la mayor movilidad que el endoscopio imprime al ojo del cirujano, proporcionan una visión panorámica del campo operatorio y brindan la posibilidad ocasionalmente única de identificar algunas estructuras o de obtener una visión más clara de ellas (protuberancias óptica y carotídea, recesos opto-carotídeos, clivus, cisternas supraselares).
La detección de fístulas de 1.c.r. se ve particularmente beneficiada por la exploración endoscópica, toda vez que permite la observación de áreas ubicadas por fuera del túnel, que el speculum determina en la exposición microquirúrgica tradicional ${ }^{19}$.

La tinción constante del extremo distal del lente por sangre con la consecuente afectación de la inspección endoscópica, ha sido señalada por varios autores ${ }^{2,9}$ y sin duda es un hecho que adiciona tiempo a la intervención. Aunque los sistemas de irrigación succión que complementan los endoscopios modernos como el que empleamos en el presente trabajo, ofrecen la posibilidad de un lavado continuo de la punta del lente y, por lo tanto, de minimizar este obstáculo. Este es uno de los detalles más molesto que debemos superar.

\section{Curación}

Jho y Carrau, en un reporte de sus primeras experiencias en 1997 refieren haber logrado resolución inicial de los síntomas en 13 de 14 adenomas intervenidos de forma endoscópica $^{20}$. Heilman et al., lograron exéresis total en 2 de 5 pacientes con adenoma hipofisario mediante el mencionado abordaje y mejoría sintomática y hormonal en otros 2, mientras que en 1 con enfermedad de Cushing se mantuvieron elevados los niveles de cortisol $^{15}$.

Los resultados obtenidos en el presente estudio de los pacientes con adenomas se asemejan a los de los autores previamente mencionados y a los que obtenemos mediante el abordaje microquirúrgico estándar, a pesar de ser la experiencia y habilidades con el abordaje endoscópico considerablemente limitadas en nuestras manos, si lo comparamos con éste. Las series de adenomas intervenidos por vía transeptoesfenoidal microquirúrgica reportadas por diversos autores ${ }^{2,9}$, no superan tampoco en general, en lo concerniente a la curación, a los que observamos en nuestra pequeña serie. Las observaciones en cuestión resultan alentadoras para el futuro desarrollo de esta técnica.

Con respecto a las fístulas, los resultados fueron menos alentadores y el hecho de que los dos pacientes en los que se mantuvo la rinorrea, fueron precisamente aquellos en los cuales la salida de 1.c.r no se observó durante la exploración endoscópica nos sugiere que otros detalles técnicos como la no utilización de fluoresceína pudieron haber influido.

\section{Morbilidad}

La única complicación que repercutió en la evolución postoperatorio fue la meningoencefalitis rápidamente controlada con antibióticos en 1 semana, que se presentó en un caso intervenido para ocluir una fístula de 1.c.r. A este caso se le colocó un drenaje espinal continuo que se mantuvo en 
el postoperatorio y la infección del sistema nervioso se hizo sintomática al cuarto día. No existen elementos para afirmar que el corredor de trabajo es menos estéril en el abordaje endoscópico que en el microquirúrgico, ya que sus estructuras anatómicas y flora bacteriana, son básicamente las mismas en ambos y su transgresión quirúrgica menor. Nos queda la interrogante del papel que el catéter espinal podría haber desempeñado como puerta de entrada en el origen de la sepsis. Las otras complicaciones que reportamos consistieron en salida de 1.c.r. a través del piso selar en 2 pacientes durante el postoperatorio inmediato, que fueron controladas durante esta misma etapa mediante las medidas habituales de reparación del mismo y colocación de un drenaje espinal continuo por 72 horas.

La diabetes insípida, complicación nada infrecuente en la cirugía hipofisaria, no se presentó en nuestros casos.

Las complicaciones propias del traumatismo quirúrgico de la vía, que con alguna frecuencia se observan en la operación transeptoesfenoidal microscópica, tales como: sinequias consecutivas a desgarros de la mucosa nasal, trastornos sensitivos gingivales o en labio superior y/o cambio de coloración de incisivos superiores, estuvieron ausentes en nuestra casuística; tampoco se observó la inflamación nasofacial postoperatoria, secundaria a la disección septal con desinserción del tabique cartilaginoso, exéresis del tabique óseo y colocación del espéculo, lo que unido a la ausencia de sondas en las fosas nasales, hacen que el postoperatorio sea más confortable.

Utilizando este mismo proceder, Cappabianca encontró que un mes después de la cirugía, la mucosa nasal estaba íntegra en todos sus casos. Tres días después de la operación solamente 5 pacientes de 15 presentaban cefalea, la cual se controlaba bien con dosis bajas de analgésicos habituales. Ninguno de sus casos necesitó taponamiento nasal. Uno de sus pacientes tuvo rinitis bacteriana que resolvió con antibióticos apropiados. Hubo, además, 3 casos con diabetes insípida transitoria que se controló en menos de 72 horas y un caso con parálisis del tercer y sexto pares craneales reversible al mes de la operación. Este autor concluye diciendo que el curso postoperatorio en general es excelente y se minimizan las dificultades respiratorias inmediatas en comparación con el método microquirúrgico ${ }^{4}$. Heilman, reporta 2 fístulas y una diabetes insípida en $9 \operatorname{casos}^{15}$. Papay y su grupo afirman que el abordaje transnasal transeptal endoscópico tiene menor riesgo de sangrado operatorio y menor morbilidad que el abordaje microquirúrgico ${ }^{27}$.

Aunque no tuvimos, en este estudio, ningún caso operado previamente de forma sublabial transeptal transesfenoidal, por observaciones propias y de numerosos autores, sabemos que en estos pacientes las reintervenciones son usualmente muy laboriosas y de mayor riesgo, pues el cirujano se enfrenta a una anatomía distorsionada, donde la mucosa septal puede tener sinequias y cicatrices, partes del septo pueden estar perforadas o ausentes, la adherencia cicatrizal entre las mucosas de ambas fosas nasales donde el tabique falta, hacen su disección muy tediosa y la conservación de su integridad muy difícil; puede haber mucoceles postoperatorios y fibrosis intraselar ${ }^{27}$. Estas condiciones pueden favorecer una orientación anatómica errónea, la pérdida de identificación de la línea media y mayor riesgo de lesión alrededor y dentro de la silla ${ }^{28}$.

En un caso operado previamente por el método microquirúrgico y reintervenido por Heilman mediante la técnica endoscópica debido a recurrencia tumoral, este autor constató que el segundo abordaje produjo menos dolor y molestias postoperatorias que el primero ${ }^{15}$.

A pesar de no estar todavía en un punto de consolidación similar al alcanzado por el abordaje microquirúrgicola morbilidad del método endoscópico es hoy inferior a la mostrada por aquel del cual existe una basta experiencia universal. Esto le augura un futuro promisorio en relación con este aspecto.

\section{Tiempo quirúrgico}

El tiempo quirúrgico promedio para el abordaje microquirúrgico en nuestro servicio se sitúa alrededor de las 2 horas, inferior al que consumimos con el método endoscópico. La explicación es obvia. En uno tenemos experiencia y habilidades desarrolladas durante años, lo que no ocurre con el otro. En este sentido encontramos en la literatura varias reflexiones.

Carrau y Jho, reconocen una curva de aprendizaje prolongada para el cirujano no familiarizado con la técnica endoscópica, dado que el método microquirúrgico y el endoscópico requieren habilidades quirúrgicas diferentes y plantean que un cirujano no experimentado se enfrentará a serias frustraciones, al tener que desplazar ambos instrumentos a través de un corredor estrecho. La introducción de endoscopios estereoscópicos en el futuro, probablemente, mejorará este problema, así como las prácticas en el laboratorio de anatomía y el uso del endoscopio durante el abordaje microquirúrgico tradicional, para comparar la visión con ambas técnicas ${ }^{6}$.

Para facilitar la operación, la escuela de Nápoles ha desarrollado una serie de instrumentos con mayor maniobrabilidad con respecto a las estructuras anatómicas atravesadas, que confieren mayor seguridad a la misma ${ }^{5}$.

Papay et al., aseguran que el sacrificio de la visión binocular, el control manual constante del endoscopio y la falta de adiestramiento suficiente son factores que conspiran contra un abordaje seguro y rápido, pero una vez adquiridas todas las habilidades necesarias el tiempo quirúrgico puede llevarse a cabo en 40 minutos ${ }^{27}$.

Resultan alentadoras para nuestra limitada experiencia 
las reflexiones que los pioneros en este abordaje han publicado al respecto.

\section{Estancia hospitalaria}

En nuestros casos la estancia hospitalaria media fue prácticamente igual en los dos métodos; sin embargo, tenemos que señalar que un paciente fue dado de alta a los dos días de la intervención, lo que nunca fue posible con el proceder microquirúrgico. Otros tres pacientes a los que se les dio el alta, uno al cuarto y dos al quinto día, en realidad estaban listos para ser dados de alta desde antes, pero se mantuvieron ingresados para evaluar de cerca su evolución, dada la escasez de experiencia con el nuevo método.

Incrementaron la estancia dos casos con fístulas transoperatorias a los que se les colocó drenaje espinal continuo durante cinco días y reposo absoluto en cama; uno de ellos desarrolló meningoencefalitis, lo que contribuyó a retrasar su alta. Los dos casos operados por fístula de LCR que no resolvieron con el tratamiento permanecieron en la sala 10 y 13 días respectivamente.

Heilman reporta una estancia postoperatoria entre 1 y 6 días, logrando dar de alta a 3 pacientes el primer día y 2 el segundo entre 9 operados. Para este autor, aquellos pacientes que no hayan tenido fístula de 1.c.r. peroperatoria, pueden ser dados de alta hospitalaria al siguiente día de la intervención ${ }^{15}$.

Los antecedentes enumerados nos sugieren que una estancia posoperatoria segura pudiera estar alrededor de las 72 horas en los casos no complicados, con su consiguiente impacto económico positivo. El ganar en experiencia en la operación y en el seguimiento sistemático, debe aumentar la confianza en los resultados y podría reducir aún más la estancia hospitalaria.

\section{Limitaciones}

Como han planteado otros autores ${ }^{4} \mathrm{y}$ hemos mencionado previamente, existen varias limitaciones relacionadas con el abordaje:

1) El endoscopio necesita un campo quirúrgico con el mínimo de sangre para hacer viable el procedimiento.

2) Aunque la visión global obtenida con el endoscopio es más amplia y abarcadora, mostrando una perspectiva panorámica de la anatomía, el cirujano carecerá de las ventajas de la visión tridimensional a lo que deberá acostumbrarse.

3) La identificación de la línea media en los casos en que el seno esfenoidal no esté simétricamente dividido por un tabique único, variante por demás infrecuente, es más difícil que con el uso del abordaje transeptal microquirúrgico estándar, lo que solo podrá ser superado con la experiencia, a menos que se cuente con sis- tema de neuronavegación.

4) La mejor tecnología disponible para hacer la intervención menos azarosa es aún cara y en franco desarrollo, por lo que la operación frecuentemente se realiza con el instrumental diseñado para cirugía otorrinolaringológica o transesfenoidal convencional.

\section{Consideraciones finales}

A pesar de las aparentes ventajas del método, importantes personalidades internacionales en este campo, las cuales no citamos por consideraciones éticas, no son partidarias del mismo.

Los resultados obtenidos en la fase 1 del presente ensayo con la utilización del método transnasal endoscópico, justifican el pase a la fase II del mismo (aleatorizada y controlada), en busca de información más consistente, en cuanto al valor real de la técnica a estas alturas del desarrollo de la cirugía hipofisaria.

\section{Bibliografía}

1. Annegers, J.F., Coulam, C.B., Abboud, C.F., Laws, E.R., Jr., Kurland, L.T.: Pituitary adenoma in Olmsted County, Minnesota, 1935-1977: a report of an increasing incidente of diagnosis in women of childbearing age. Mayo Clin Proc 1978; 53 : 641-643.

2. Becker, P., Schaehter, D., Gallardo, E., Colin, E., Selman, J., Martínez, C.: Pituitary tumors: clinical presentation and evaluation of transphenoidal surgical treatment. Rev Med Chil. 1994 Jul; 122: 769-776.

3. Bushe, K.A., Halves, E.: Modified technique in transsphenoidal operations of pituitary adenomas: Technical note [in German]. Acta Neurochir 1978; 41: 163-175.

4. Cappabianca, P., Alfieri, A., de Divitis, E.: Endoscopic endonasal transsphenoidal approach to the sella: towards functional endoscopio pituitary surgery (FEPS). Minim Invas Neurosurg 1998; 41: 66-73.

5. Cappabianca, P., Alfieri, A., Thermes, S., Buonamassa, S., Divitis, E.: Instruments for endoscopio endonasal transsphenoidal surgery. Neurosurgery 1999; 45: 392-397.

6. Carrau, R.L., Jho, H.D., Ko, Y.: Transnasal-transsphenoidal endoscopio surgery of the pituitary gland. Laryngoscope 1996; 106: 914-918.

7. Castillo, L., Jaklis, A., Paquis, P., Haddad, A., Santini, J.: Nasal endoscopio repair of cerebroespinal fluid rhinorrhea. Rhinology 1999; 37: 33-36.

8. Cawley, C., Tindall, G.: Transsphenoidal surgery: operative techniques. En: Krisht, A., Tindall, A., eds. Pituitary disorders: comprehensive management. Baltimore: Lippincott, Williams and Wilkins 1999: 349-359.

9. Cirio, I., Mikhael, M., Stafford, T., Lawson, L., Garces, R.: Transsphenoidal microsurgery of pituitary macroadenomas 
with long-term follow-up results. J Neurosurg. 1983 Sep; 59: 395-401.

10. Collins, W.F.: Adenomas de la hipófisis. Epidemia? Clin Quirurg Norte Am 1980; 5:1217.

11. Costello, R.T.: Subclinical adenoma of the pituitary gland. Am J Pathol 1936; 12: 205-216.

12. Gamea, A., Fathi, M., El-Guindy, A.: The use of the rigid endoscope in transsphenoidal pituitary sugery. J Laryngol Otol 1994; 108: 19-22.

13. Goldhahn, W.E.: Experience with paraorbital transethmoidal procedure in pituitary tumors[in German]. Zentralbl Neurochir 1980; 41: 177-184.

14. Griffith, H.B., Veerapen, R.: A direct transnasal approach to the sphenoid sirius. Technical note. J Neurosurg 1987; 66: $140-142$.

15. Heilman, C.B., Shucart, W.A., Rebeiz, E.E.: Endoscopic sphenoidotomy approach to the sella. Neurosurgery 1997; 41: 602-607.

16. Hirsch, O.: Endonasal method of removal of hypophyseal tumors. JAMA 1910; 5: 772-774.

17. Jane, J.A., Laws, E.R.: The surgical management of pituitary adenomas in a series of 3093 cases. J Am Coll Surg 2001; 193: 651-659.

18. Jankowski, R., Aunque, J., Simon, C., Marchal, J.C., Hepner, H., Wayoff, M.: Endoscopic pituitary tumor surgery. Laryngoscope 1992; 102: 198-202.

19. Jho, H.D., Carrau, R.L., Ko, Y.: Endoscopic pituitary surgery. En: Wilkins RH, Renganchary SS., eds. Neurosurgical operative atlas. Park Ridge 111: Am.

20. Jho, H.D., Carrau, R.L., Daly, M.A.: Endoscopic pituitary surgery: an early experience. Surg Neurol 1997; 47: 213223.

21. Jho, H.D., Carrau, R.L.: Endoscopic endonasal transsphenoidal surgery: experience with 50 patients. J Neurosurg 1997; 87: 44-51.

22. Kennedy, D.W., Zenrich, J., Rosebaum, A.M., Johns, M.E.: Functional endoscopic sinus surgery: theory and diagnostic evaluation. Arch Otolaryngol Head Neck Surg 1985; 111: 576-582.

23. Koren, I., Hadar, T., Rappaport, Z.H., Yaniv, E.: Endoscopic transnasal transsphenoidal microsurgery versus the sublabial approach for the treatmentof pituitary tumors: endonasal complications. Laryngoscope 1999; 109: 18381840
24. Lubke, D., Saeger, W.: Carcinomas of the pituitary: definition and review of the literature. Gen Diagn Pathol 1995; 141: 81-92.

25. Morita, A.: Endoscopic transnasal transesfenoidal approach to the pituitary lesions. No Shinkei Geka 1999; 27 : 699-710.

26. Papay, F.A., Benninger, M.S., Levine, H.L., et al.: Transnasal transseptal endoscopic repair of sphenoidal cerebral spinal fluid fistula. Otolaryngol Head Neck Surg 1989; 101: 595-597.

27. Papay, F.A., Stein, J.M., Rhoten, R.L.P., Luciano, M., Zins, J., Hahn, J.: Transnasal transseptal endoscopic approach to the sphenoid sinus. J Craniofacial Surg 1997; 8: 159-163.

28. Rodas, Y.E.: Complicaciones de la vía transeptoesfenoidal. Tesis de grado. Ciudad de La Habana: Hospital Hermanos Ameijeiras 1998.

29. Rodziewicz, G.S., Kelley, R.T., Kellman, R.M., Smith, M.V.: Transnasal endoscopic surgery of the pituitary gland: technical note. Neurosurgery 1996; 39: 189-198.

30. Sethi, D.S., Pillay, P.K.: Endoscopic pituitary surgery: a minimally invasive technique. Am J Rhinol 1996; 10: 141148.

31. Sheehan ,M.T., Atkison, J.L., Kasperbauer, J.L., Ericson, B.J., Nippoldt, T.B.: Preliminary comparison of the endoscopic transnasal vs the sublabial transeptal approach for clinicaly nonfuncioning pituitary macroadenomas. Mayo Clin Proc 1999; 74: 661-670.

32. Tomita, T., Gates, E.: Pituitary adenomas and granular cell tumors: incidence, cell type, and location of tumor in 100 pituitary glands at autopsy. Am J Clin Pathol 1999; 111: 817825 .

33. Tucker, H.M., Hahn J,F.: Transnasal-transseptal sphenoidal approach to hypophysectomy. Laryngoscope 1996; 106: 914-918.

González-González, J.L.; López-Arbolay, O.; Morales-Sabina, O.; Martí-Piñeiro, J.F.; Vidal-Verdial R.: Cirugía transnasal transesfenoidal endoscópica en afecciones de región selar. Neurocirugía 2005; 16: 27-23.

Correspondencia postal: Justo L. González González. Servicio de Neurocirugía. Hospital "Hermanos Ameijeiras" San Lázaro 701 Código Postal 10300. Centro Habana. Ciudad de la Habana. Cuba. 